\title{
COMPARISON AND DISJOINT-OCCURRENCE INEQUALITIES FOR RANDOM-CLUSTER MODELS
}

\author{
Geoffrey Grimmett
}

\begin{abstract}
A principal technique for studying percolation, (ferromagnetic) Ising, Potts, and random-cluster models is the FKG inequality, which implies certain stochastic comparison inequalities for the associated probability measures. The first result of this paper is a new comparison inequality, proved using an argument developed in Refs. 2, 6, 14, and 21 in order to obtain strict inequalities for critical values. As an application of this inequality, we prove that the critical point $p_{c}(q)$, of the random-cluster model with cluster-weighting factor $q(\geq 1)$, is strictly monotone in $q$. Our second result is a 'BK inequality' for the disjoint occurrence of increasing events, in a weaker form than that available in percolation theory.
\end{abstract}

\section{Introduction}

In studying ferromagnetic Ising and Potts models, it is common to concentrate in part on the associated random-cluster model (or 'Fortuin-Kasteleyn representation'). Random-cluster models have proved to be the natural setting for many arguments of value, as well as being objects worthy of study in their own right. The relationship between random-cluster models and ferromagnetic systems was first described by Fortuin and Kasteleyn, ${ }^{(8,9,10,11,19)}$ and more recently by Edwards and Sokal. ${ }^{(7)}$ For recent accounts of the theory of random-cluster models, see Refs. $1,14,15,16,17$.

The random-cluster model on a graph $G$ has two real parameters, being an 'edge-parameter' $p$ satisfying $0 \leq p \leq 1$, and a 'cluster-weighting factor' $q$ satisfying $q>0$. When $q \geq 1$, the associated probability measure satisfies the FKG lattice condition (see Refs. 1, 12), and therefore satisfies the FKG inequality (which may be stated roughly as 'increasing events are positively correlated'). This useful inequality implies properties of stochastic comparison which are of substantial value in studying any associated phase transition. The FKG inequality is generally invalid when $q<1$, and this is a principal obstacle in studying this case (see Ref. $15)$.

The primary purpose of the present note is to prove a new comparison inequality for random-cluster measures with $q \geq 1$; we do not know how to derive this inequality from an argument of FKG-type. The secondary purpose is to state and

1991 Mathematics Subject Classification. 60K35, 82B20, 82B43.

Key words and phrases. Random-cluster model, Ising model, Potts model, comparison inequality, BK inequality, FKG inequality.

Address of author. Statistical Laboratory, University of Cambridge, 16 Mill Lane, Cambridge CB2 1SB, UK. 
prove a BK inequality for random-cluster models with $q \geq 1$; this is a generalisation of the usual BK inequality for percolation, but its form for general $q$ is necessarily weaker than for the case $q=1$.

When $q \geq 1$, one may construct random-cluster measures on the infinite grid $\mathbb{Z}^{d}$, by passing to a thermodynamic limit (see Refs. 1, 15). Such measures have a phase transition at a critical value $p=p_{c}(q)$ that marks the onset of an infinite cluster (or connected component) of the graph. It is immediate from the standard comparison inequalities that $p_{c}(q) \leq p_{c}\left(q^{\prime}\right)$ if $q<q^{\prime}$, and it is natural to conjecture that strict inequality holds here. This may indeed be proved, by applying the comparison inequality proved herein. Indeed, one may go slightly further and calculate a strictly positive lower bound for the difference $p_{c}\left(q^{\prime}\right)-p_{c}(q)$ when $1 \leq q<q^{\prime}$.

The argument laid down in Ref. 2, and developed further in Refs. 6 and 14 and in this paper, has implications for the values of certain critical exponents. In studying the nature of the phase transition, it is usual to assume that $q$ is fixed, and that $p$ is near the critical point $p_{c}(q)$. However, one may think of $q$ as just another parameter, which may be varied. One is then led to study the nature of the critical point $\left(p_{c}(q), q\right)$ in $(p, q)$-space. Using the results of this paper, in the manner described in Ref. 6, it emerges that certain families of critical exponents have common values in this enhanced parameter space. We shall return briefly to this point in Section 3.

Finally we recall the relationship between Potts models and random-cluster measures. We express the finite graph $G$ as $G=(V, E)$ where $V$ is the vertex set and $E$ is the edge set; we define the state spaces $\Sigma=\{1,2, \ldots, q\}^{V}$ and $\Omega=\{0,1\}^{E}$, where $q$ is a positive integer $(q \geq 2)$. The Potts probability measure with ferromagnetic interaction $J(>0)$ and inverse-temperature $\beta$ is given by

$$
\pi(\sigma)=\frac{1}{Z} \exp \left\{-\beta J \sum_{\langle u, v\rangle}\left(1-\delta_{\sigma(u), \sigma(v)}\right)\right\}, \quad \sigma \in \Sigma,
$$

where $Z$ is the normalising factor, the sum is over all edges $\langle u, v\rangle \in E$, and $\delta_{i j}$ is the Kronecker delta,

$$
\delta_{i j}= \begin{cases}1 & \text { if } i=j \\ 0 & \text { if } i \neq j .\end{cases}
$$

The corresponding random-cluster (probability) measure is

$$
\phi_{p, q}(\omega)=\frac{1}{Z_{p, q}}\left\{\prod_{e \in E} p^{\omega(e)}(1-p)^{1-\omega(e)}\right\} q^{k(\omega)}, \quad \omega \in \Omega
$$

where

$$
Z_{p, q}=\sum_{\omega \in \Omega}\left\{\prod_{e \in E} p^{\omega(e)}(1-p)^{1-\omega(e)}\right\} q^{k(\omega)}
$$

is the appropriate normalising factor, and where

$$
p=1-e^{-\beta J},
$$

and $k(\omega)$ is the number of connected components of the graph with vertex set $V$ and edge set $\{e \in E: \omega(e)=1\}$. 
One may obtain Potts and random-cluster measures on the infinite lattice $\mathbb{Z}^{d}$ by passing to appropriate thermodynamic limits (see Refs. 1, 17). It turns out that the critical inverse-temperature $\beta_{c}=\beta_{c}(J, q)$ of the Potts model is related by (1.3) to the critical value $p_{c}(q)$ of the associated random-cluster model. Therefore the strict monotonicity of $p_{c}(q)$, as $q$ increases, implies the strict monotonicity of $\beta_{c}$ as a function of $q$.

In Section 2 we present the new comparison inequality, and we apply it in Section 3. Section 4 contains the BK inequality for random-cluster models satisfying the FKG inequality.

\section{Comparison inequalities}

Let $G=(V, E)$ be a finite graph, and suppose that $0 \leq p \leq 1$ and $q>0$. The appropriate state space is the set $\Omega=\{0,1\}^{E}$ of all vectors $\omega=(\omega(e): e \in E)$ with zero/one entries. We call an edge e open (in $\omega$ ) if $\omega(e)=1$, and we write $\eta(\omega)=\{e: \omega(e)=1\}$ for the set of open edges. The random-cluster measure $\phi_{p, q}$ on $G$ with parameters $p$ and $q$ is defined by (1.1) and (1.2).

It is well known ${ }^{(1,17)}$ that $\phi_{p, q}$ satisfies the FKG inequality if $q \geq 1$. Here is some notation prior to the statement of this inequality. We call a function $f: \Omega \rightarrow \mathbb{R}$ increasing if $f(\omega) \leq f\left(\omega^{\prime}\right)$ whenever $\omega \leq \omega^{\prime}$ (i.e., whenever $\omega(e) \leq \omega^{\prime}(e)$ for all $e \in E) ; f$ is called decreasing if $-f$ is increasing. For a random variable $f$ and a probability measure $\mu$ on $\Omega$, we denote by $\mu(f)$ the mean of $f$ under $\mu$,

$$
\mu(f)=\sum_{\omega \in \Omega} f(\omega) \mu(\omega) .
$$

Theorem 2.1 (FKG inequality). If $q \geq 1$, then

$$
\phi_{p, q}(f g) \geq \phi_{p, q}(f) \phi_{p, q}(g)
$$

for all increasing functions $f, g: \Omega \rightarrow \mathbb{R}$.

Given two probability measures $\mu_{1}$ and $\mu_{2}$ on $\Omega$, we say that $\mu_{2}$ dominates $\mu_{1}$, written $\mu_{1} \leq \mu_{2}$, if $\mu_{1}(f) \leq \mu_{2}(f)$ for all increasing $f: \Omega \rightarrow \mathbb{R}$. The FKG inequality enables the proof of two standard comparison inequalities for randomcluster measures.

Theorem 2.2 (Comparison inequalities). It is the case the

$$
\begin{aligned}
& \phi_{p^{\prime}, q^{\prime}} \leq \phi_{p, q} \quad \text { if } \quad q^{\prime} \geq q, \quad q^{\prime} \geq 1, p^{\prime} \leq p, \\
& \phi_{p^{\prime}, q^{\prime}} \geq \phi_{p, q} \quad \text { if } \quad q^{\prime} \geq q, \quad q^{\prime} \geq 1, \quad \frac{p^{\prime}}{q^{\prime}\left(1-p^{\prime}\right)} \geq \frac{p}{q(1-p)} .
\end{aligned}
$$

Although these were first proved by Fortuin and Kasteleyn, we refer the reader to Refs. 1 and 17 for modern accounts. The next theorem is our new result, which extends (2.3). For a reason which will become evident in Section 3, we need to introduce another piece of terminology. A subset $S$ of the vertex set $V$ is called spanning if every edge of $E$ is incident to at least one vertex of $S$. The degree $\operatorname{deg}(S)$ of a spanning set $S$ is defined to be the maximum degree of its members, i.e., the maximum number of edges incident to any one vertex in $S$. We assume henceforth that $q \geq 1$. 
Theorem 2.3. (a) For each $\delta>0$, there exists a continuous function $\alpha(p, q)=$ $\alpha(p, q, \delta)$, which is strictly positive when $0<p<1, q \geq 1$, such that the following holds. If there exists a spanning set $S$ with $\operatorname{deg}(S) \leq \delta$, then

$$
\alpha(p, q) \frac{\partial}{\partial p} \phi_{p, q}(A) \leq-q \frac{\partial}{\partial q} \phi_{p, q}(A) \leq p(1-p) \frac{\partial}{\partial p} \phi_{p, q}(A)
$$

for all increasing events $A$.

(b) For each $\delta>0$, there exists a continuous function $\gamma(p, q)=\gamma(p, q, \delta)$, which is strictly increasing in $p$ on $[0,1]$, and strictly decreasing in $q$ on $[1, \infty)$, such that the following holds. If there exists a spanning set $S$ with $\operatorname{deg}(S) \leq \delta$, then

$$
\phi_{p^{\prime}, q^{\prime}} \leq \phi_{p, q} \quad \text { if } \quad 1 \leq q \leq q^{\prime} \text { and } \gamma\left(p^{\prime}, q^{\prime}\right) \geq \gamma(p, q) \text {. }
$$

We note that (2.3) implies

$$
\frac{\partial}{\partial q} \phi_{p, q}(A) \leq 0 \leq \frac{\partial}{\partial p} \phi_{p, q}(A) \text { for all increasing } A \text {. }
$$

The function $\gamma(p, q)$ in Theorem 2.3(b) may be taken as any function which satisfies the partial differential equation

$$
\alpha(p, q) \frac{\partial \gamma}{\partial p}+q \frac{\partial \gamma}{\partial q}=0, \quad 0<p<1, q>1
$$

subject to

$$
\frac{\partial \gamma}{\partial q}<0<\frac{\partial \gamma}{\partial p}, \quad 0<p<1, q>1
$$

Proof of Theorem 2.3. (a) Let $A$ be an increasing event, and write $\theta(p, q)=\phi_{p, q}(A)$. We prove (2.5) following the scheme laid down in Ref. 2 and developed in Refs. 6 and 14 . The method proceeds by constructing a Markov chain $Z_{t}=\left(X_{t}, Y_{t}\right)$, taking values in the state space $\Omega^{2}$. We shall not give full details of this construction, but refer to Ref. 6 for further discussion where necessary.

For $\omega \in \Omega$ and $f \in E$, we define the configurations $\omega_{f}$ and $\omega^{f}$ by

$$
\omega_{f}(g)=\left\{\begin{array}{ll}
\omega(g) & \text { if } g \neq f, \\
0 & \text { if } g=f,
\end{array} \quad \omega^{f}(g)= \begin{cases}\omega(g) & \text { if } g \neq f \\
1 & \text { if } g=f\end{cases}\right.
$$

Let $D_{f}(\omega)$ be the indicator function of the event that the endpoints of $f$ are in different components of the graph $\left(V, \eta\left(\omega_{f}\right)\right)$, i.e., the event that the endpoints of $f$ are connected by no open path that does not use $f$ itself. We define the functions $H, H^{A}: \Omega^{2} \rightarrow \mathbb{R}$ as follows. First,

$$
\begin{aligned}
& H\left(\omega_{f}, \omega^{f}\right)=1, \\
& H\left(\omega^{f}, \omega_{f}\right)=\frac{1-p}{p} q^{D_{f}(\omega)},
\end{aligned}
$$


for $\omega \in \Omega$ and $f \in E$. Secondly, $H\left(\omega, \omega^{\prime}\right)$ is set to 0 for other pairs $\omega, \omega^{\prime}$ with $\omega \neq \omega^{\prime}$. Next, we define $H^{A}$ by

$$
H^{A}\left(\omega, \omega^{\prime}\right)=H\left(\omega, \omega^{\prime}\right) I_{A}\left(\omega \wedge \omega^{\prime}\right) \text { if } \omega \neq \omega^{\prime}
$$

where $I_{A}$ is the indicator function of $A$, and $\omega \wedge \omega^{\prime}(g)=\min \left\{\omega(g), \omega^{\prime}(g)\right\}$. The diagonal terms $H(\omega, \omega)$ and $H^{A}(\omega, \omega)$ are chosen in such a way that

$$
\sum_{\omega^{\prime} \in \Omega} H\left(\omega, \omega^{\prime}\right)=\sum_{\omega^{\prime} \in \Omega} H^{A}\left(\omega, \omega^{\prime}\right)=0 \quad \text { for all } \omega \in \Omega .
$$

Now we are ready to define the chain $Z_{t}$. Let $J: \Omega^{2} \times \Omega^{2} \rightarrow \mathbb{R}$ be given by

$$
\begin{aligned}
J\left(\pi_{f}, \omega ; \pi^{f}, \omega^{f}\right) & =1, \\
J\left(\pi, \omega^{f} ; \pi_{f}, \omega_{f}\right) & =H^{A}\left(\omega^{f}, \omega_{f}\right), \\
J\left(\pi^{f}, \omega^{f} ; \pi_{f}, \omega^{f}\right) & =H\left(\pi^{f}, \pi_{f}\right)-H^{A}\left(\omega^{f}, \omega_{f}\right),
\end{aligned}
$$

for all $(\pi, \omega) \in \Omega^{2}$ satisfying $\pi \leq \omega$, and for all $f \in E$. All other off-diagonal values of $J$ are set to 0 , and the diagonal elements are chosen so that

$$
\sum_{\left(\pi^{\prime}, \omega^{\prime}\right)} J\left(\pi, \omega ; \pi^{\prime}, \omega^{\prime}\right)=0 \text { for all } \pi, \omega
$$

The function $J$ will be used as the generator of a Markov chain on the state space $\Omega^{2}$. With $J$ viewed in this way, equation (2.12) specifies that, for $\pi \in \Omega$ and $f \in E$, the edge $f$ is acquired by $\pi$ (if it does not already contain it) at rate 1 ; any edge so acquired is added also to $\omega$ if it does not already contain it. Equation (2.13) specifies that, for $\omega \in \Omega$ and $f \in \eta(\omega)$, the edge $f$ is removed from $\omega$ (and also from $\pi$ if $f \in \eta(\pi))$ at the rate given by (2.11). For $f \in \eta(\pi)(\subseteq \eta(\omega))$, there is an additional rate at which $f$ is removed from $\pi$ but not from $\omega$. Note that this additional rate is indeed non-negative, since

$$
H\left(\pi^{f}, \pi_{f}\right)-H^{A}\left(\omega^{f}, \omega_{f}\right)=\frac{1-p}{p}\left\{q^{D_{f}(\pi)}-q^{D_{f}(\omega)} I_{A}\left(\omega_{f}\right)\right\} \geq 0,
$$

by (2.10) and (2.11); remember that $q \geq 1$ and $D_{f}(\omega) \leq D_{f}(\pi)$ when $\pi \leq \omega$.

It is shown in Ref. 6 that there exists a Markov chain $Z_{t}=\left(X_{t}, Y_{t}\right)$, on $\Omega^{2}$, such that

(i) $Z_{t}$ has generator $J$ [i.e., for $(\pi, \omega) \neq\left(\pi^{\prime}, \omega^{\prime}\right), J\left(\pi, \omega ; \pi^{\prime}, \omega^{\prime}\right)$ represents the jump-rate of the chain from $(\pi, \omega)$ to $\left.\left(\pi^{\prime}, \omega^{\prime}\right)\right]$,

(ii) the limiting measure of $X_{t}$, as $t \rightarrow \infty$, is $\phi_{p, q}(\cdot)$,

(iii) the limiting measure of $Y_{t}$, as $t \rightarrow \infty$, is $\phi_{p, q}(\cdot \mid A)$,

(iv) $X_{t} \leq Y_{t}$ for all $t$.

Evidently

$$
\phi_{p, q}(A)=\frac{1}{Z_{p, q}} \sum_{\omega \in \Omega} I_{A}(\omega)\left\{\prod_{f \in E} p^{\omega(f)}(1-p)^{1-\omega(f)}\right\} q^{k(\omega)} .
$$


Differentiating with respect to $p$, one obtains, as in Ref. 6 , that

$$
\begin{aligned}
\frac{\partial \theta}{\partial p}=\frac{1}{p(1-p)} \operatorname{cov}\left(N, I_{A}\right) & =\frac{\theta(p, q)}{p(1-p)}\left\{\lim _{t \rightarrow \infty} P\left(N\left(Y_{t}\right)-N\left(X_{t}\right)\right)\right\} \\
& =\frac{\theta(p, q)}{p(1-p)} \sum_{e \in E} \lim _{t \rightarrow \infty} P\left(X_{t}(e)=0, Y_{t}(e)=1\right),
\end{aligned}
$$

where $N=N(\omega)=|\{e: \omega(e)=1\}|$, 'cov' denotes covariance with respect to $\phi_{p, q}$, and $P$ is the appropriate probability measure for the chain $Z_{t}$. A similar elementary calculation yields that

$$
\frac{\partial \theta}{\partial q}=\frac{1}{q} \operatorname{cov}\left(k, I_{A}\right)=-\frac{1}{q} \theta(p, q)\left\{\lim _{t \rightarrow \infty} P\left(k\left(X_{t}\right)-k\left(Y_{t}\right)\right)\right\},
$$

where $k=k(\omega)$ is the number of components of the graph $(V, \eta(\omega))$ as usual.

By an elementary graph-theoretic argument,

$$
k\left(X_{t}\right)-k\left(Y_{t}\right) \leq N\left(Y_{t}\right)-N\left(X_{t}\right),
$$

whence

$$
-q \frac{\partial \theta}{\partial q} \leq p(1-p) \frac{\partial \theta}{\partial p}
$$

which is the right-hand inequality of (2.5).

Let $\delta$ be a positive integer, and suppose that $S$ is a spanning set of vertices satisfying $\operatorname{deg}(S) \leq \delta$. For $x \in V$, let $I_{x}$ be the indicator function of the event that $x$ is an isolated vertex. Clearly

$$
P\left(k\left(X_{t}\right)-k\left(Y_{t}\right)\right) \geq \sum_{x \in S} P\left(I_{x}\left(X_{t}\right)=1, I_{x}\left(Y_{t}\right)=0\right),
$$

since the right-hand side counts the number of vertices of $S$ which are isolated in $X_{t}$, but which are not isolated in $Y_{t}$. Let $x \in S$, and let $e_{x}$ be any edge of $E$ which is incident to $x$. We claim that

$$
\nu P\left(X_{t}\left(e_{x}\right)=0, Y_{t}\left(e_{x}\right)=1\right) \leq P\left(I_{x}\left(X_{t+1}\right)=1, I_{x}\left(Y_{t+1}\right)=0\right)
$$

for some $\nu=\nu(p, q)$ which is continuous, and is strictly positive on $(0,1) \times[1, \infty)$; here, $\nu$ is allowed to depend on the value of $\delta$ but not further upon $x, e_{x}, S$, or $G$. Once (2.20) is proved, the left-hand inequality of (2.5) follows immediately by summing (2.20) over $x$ and using (2.17)-(2.19) as follows:

$$
\begin{aligned}
-q \frac{\partial \theta}{\partial q} & \geq \theta\left\{\lim _{t \rightarrow \infty} \sum_{x \in S} P\left(I_{x}\left(X_{t+1}\right)=1, I_{x}\left(Y_{t+1}\right)=0\right)\right\} \\
& \geq \frac{\theta \nu}{\delta}\left\{\lim _{t \rightarrow \infty} \sum_{e \in E} P\left(X_{t}(e)=0, Y_{t}(e)=1\right)\right\} \\
& =\frac{\theta \nu}{\delta} p(1-p) \frac{\partial \theta}{\partial p} .
\end{aligned}
$$


Finally we prove (2.20). Let $E_{x}$ be the set of edges of $E$ which are incident to $x$. Suppose that the event $T_{t}=\left\{X_{t}\left(e_{x}\right)=0, Y_{t}\left(e_{x}\right)=1\right\}$ occurs. Let

(a) $U$ be the event that, during the time-interval (t,t+1), every edge $f$ of $E_{x} \backslash\left\{e_{x}\right\}$ with $X_{t}(f)=1$ changes its $X$-state from 1 to 0 ; the removal of such edges from $X$ may involve also their removal from $Y$ (see Ref. 6, Sect. 4),

(b) $V$ be the event that no edge $f$ of $E_{x} \backslash\left\{e_{x}\right\}$ with $X_{t}(f)=0$ changes its state in the time-interval $(t, t+1)$,

(c) $W$ be the event that the state of the edge $e_{x}$ remains unchanged during $(t, t+1)$.

As described in Ref. 6, there exists a strictly positive and continuous function $\nu_{S}=\nu_{S}(p, q)$ on $(0,1) \times[1, \infty)$ (which is allowed to depend on $G$ and $S$ only through the quantity $\operatorname{deg}(S))$ such that

$$
P\left(U \cap V \cap W \mid T_{t}\right) \geq \nu_{S} \quad \text { for all } t,
$$

uniformly in $x, e_{x}, G$, and $t$. This inequality remains true if we replace $\nu_{S}$ by the positive continuous function $\nu$ defined by

$$
\nu(p, q, \delta)=\min \left\{\nu_{S}(p, q): 0 \leq \operatorname{deg}(S) \leq \delta\right\} .
$$

Now, if $T_{t} \cap U \cap V \cap W$ occurs, then $x$ is isolated in $X_{t+1}$, but not isolated in $Y_{t+1}$ (since $\left.Y_{t+1}\left(e_{x}\right)=1\right)$. Therefore $(2.20)$ is valid, and the proof of (a) is complete. A function $\nu$ of the required form may be written down explicitly.

(b) Inequality (2.5) may be stated as

$$
(\alpha, q) \cdot \nabla \theta \leq 0 \leq(p(1-p), q) . \nabla \theta
$$

and we have in addition that

$$
\frac{\partial \theta}{\partial q} \leq 0 \leq \frac{\partial \theta}{\partial p}
$$

by Theorem 2.2. Possibly aided by sketching the characteristics of the partial differential equations

$$
(\alpha, q) . \nabla f=0, \quad(p(1-p), q) . \nabla f=0,
$$

we find that the right-hand inequality of (2.21), together with (2.22), implies the second part of Theorem 2.2. Similarly the left-hand inequality of (2.21) implies (2.6), with $\gamma$ a solution of the equation $(\alpha, q) . \nabla \gamma=0$ satisfying

$$
\frac{\partial \gamma}{\partial q}<0<\frac{\partial \gamma}{\partial p} \quad \text { when } 0<p<1, q>1
$$




\section{Applications}

We give two applications of Theorem 2.3 to the phase transition for random-cluster models. Here is some notation. The hypercubic lattice is denoted by $\mathbb{Z}^{d}$, with edge-set $\mathbb{E}$, and we assume $d \geq 2$. For any finite box $\Lambda$ in $\mathbb{Z}^{d}$, the boundary $\partial \Lambda$ is the set of all vertices in $\Lambda$ which are adjacent to some vertex outside $\Lambda$. The edge-set $\mathbb{E}_{\Lambda}$ is the set of all edges in $\mathbb{E}$ both of whose endpoints lie in $\Lambda$.

Let $\Omega=\{0,1\}^{\mathbb{E}}$; the appropriate $\sigma$-field $\mathcal{F}$ is generated by the finite-dimensional cylinders of $\Omega$. For a finite box $\Lambda$, we write $\Omega_{\Lambda}$ for the set of all $\omega(\in \Omega)$ satisfying $\omega(e)=1$ if $e \notin \mathbb{E}_{\Lambda}$. We call an edge $e$ open (in $\omega$ ) if $\omega(e)=1$, and we write $\eta(\omega)=\{e: \omega(e)=1\}$ as before.

Next we introduce some probability measures on $(\Omega, \mathcal{F})$. Let $\Lambda$ be a finite box, and suppose $0 \leq p \leq 1$ and $q \geq 1$. Denote by $\phi_{\Lambda, p, q}$ the probability measure with support $\Omega_{\Lambda}$ satisfying

$$
\phi_{\Lambda, p, q}(\omega)=\frac{1}{Z_{\Lambda, p, q}}\left\{\prod_{e \in \mathbb{E}_{\Lambda}} p^{\omega(e)}(1-p)^{1-\omega(e)}\right\} q^{k(\omega, \Lambda)}, \quad \omega \in \Omega_{\Lambda},
$$

where $Z_{\Lambda, p, q}$ is the normalising factor and $k(\omega, \Lambda)$ is the number of components of the infinite graph $\left(\mathbb{Z}^{d}, \eta(\omega)\right)$ which intersect $\Lambda$. It is easily seen that $\phi_{\Lambda, p, q}$ is essentially the random-cluster measure (given in (1.1)) on the graph obtained from $\mathbb{Z}^{d}$ by identifying all vertices lying outside $\Lambda \backslash \partial \Lambda$.

It is well known (see Refs. 1, 15, 17) that the weak limit

$$
\phi_{p, q}=\lim _{\Lambda \rightarrow \mathbb{Z}^{d}} \phi_{\Lambda, p, q}
$$

exists. Let 0 be the origin of $\mathbb{Z}^{d}$, and let $\{0 \leftrightarrow \infty\}$ denote the event that 0 lies in some infinite open path. It is a standard result that the critical value

$$
p_{c}(q)=\sup \left\{p: \phi_{p, q}(0 \leftrightarrow \infty)=0\right\}
$$

exists and satisfies

$$
0<p_{c}(q)<1 \quad \text { if } q \geq 1
$$

We state some properties of $p_{c}(q)$ next. Part (a) is implicit in Ref. 1.

Theorem 3.1. (a) If $1 \leq q \leq q^{\prime}$ then

$$
\frac{1}{p_{c}\left(q^{\prime}\right)} \leq \frac{1}{p_{c}(q)} \leq \frac{q^{\prime} / q}{p_{c}\left(q^{\prime}\right)}-\frac{q^{\prime}}{q}+1
$$

(b) $p_{c}(q)$ is a Lipschitz-continuous and strictly increasing function of $q$ on $[1, \infty)$.

The percolation probability $\theta(p, q)$ is defined as the probability that the origin lies in an infinite open cluster,

$$
\theta(p, q)=\phi_{p, q}(0 \leftrightarrow \infty)
$$


One of the principal critical exponents of the model is the quantity $\beta=\beta(q)$ satisfying

$$
\beta(q)=\lim _{p \downarrow p_{c}(q)}\left\{\frac{\log \left[\theta(p, q)-\theta\left(p_{c}(q), q\right)\right]}{\log \left[p-p_{c}(q)\right]}\right\},
$$

if this limit exists; this is the exponent arising from the asymptotic behaviour of $\theta(p, q)$ as $p \downarrow p_{c}(q)$. Another exponent which is natural in the random-cluster setting is

$$
\beta^{\prime}(q)=\lim _{q^{\prime} \uparrow q}\left\{\frac{\log \left[\theta\left(p_{c}(q), q^{\prime}\right)-\theta\left(p_{c}(q), q\right)\right]}{\log \left[q-q^{\prime}\right]}\right\},
$$

if this limit exists; this is the exponent related to the behaviour of $\theta\left(p_{c}(q), q^{\prime}\right)$ as $q^{\prime} \uparrow q$. It is a consequence of Theorem 2.3 that

$$
\beta^{\prime}(q)=\beta(q) \text { if } q>1 .
$$

The proof of this fact follows that of Ref. 6 , Thm 2 (see also the discussion after Eqn (2.9) of that paper). Similar conclusions may be obtained for other critical exponents, arising both from other directions of approach of the point $\left(p_{c}(q), q\right)$ and from other order parameters.

Proof of Theorem 3.1. (a) Suppose $1 \leq q \leq q^{\prime}$, and let $\Lambda$ be a finite box of $\mathbb{Z}^{d}$. It is fundamental (see Refs. 1, 15) that

$$
\theta(p, q)=\lim _{\Lambda \rightarrow \mathbb{Z}^{d}} \phi_{\Lambda, p, q}(0 \leftrightarrow \infty) .
$$

Now $\phi_{\Lambda, p, q}$ may be thought of as the random-cluster measure on the finite graph $\bar{\Lambda}$ obtained from $\Lambda$ by identifying all vertices lying in the boundary $\partial \Lambda$. By (2.3),

$$
\theta\left(p, q^{\prime}\right) \leq \theta(p, q)
$$

whence $p_{c}\left(q^{\prime}\right) \geq p_{c}(q)$. By $(2.4)$,

$$
\theta\left(p^{\prime}, q^{\prime}\right) \geq \theta(p, q) \quad \text { if } \quad \frac{p^{\prime}}{q^{\prime}\left(1-p^{\prime}\right)} \geq \frac{p}{q(1-p)} .
$$

Therefore, if $p^{\prime}<p_{c}\left(q^{\prime}\right)$, then $p<p_{c}(q)$ whenever

$$
\frac{p^{\prime}}{q^{\prime}\left(1-p^{\prime}\right)} \geq \frac{p}{q(1-p)}
$$

This implies the right-hand side of (3.2).

(b) The Lipschitz-continuity of $p_{c}(q)$ is an immediate consequence of (3.2), following easy manipulation. As for strict monotonicity, let $\gamma$ be given as in Theorem 2.3(b) with $\delta=2 d$, and let $1 \leq q<q^{\prime}$. We apply Theorem 2.3 (b) to the graph $\bar{\Lambda}$, with spanning set $S=\Lambda \backslash \partial \Lambda$ satisfying $\operatorname{deg}(S)=2 d$. It follows from (2.6) and (3.6) that

$$
\theta\left(p^{\prime}, q^{\prime}\right) \leq \theta(p, q) \quad \text { if } \quad \gamma\left(p^{\prime}, q^{\prime}\right) \geq \gamma(p, q) .
$$

This implies that

$$
\gamma\left(p_{c}\left(q^{\prime}\right), q^{\prime}\right) \leq \gamma\left(p_{c}(q), q\right)
$$

since if $\gamma\left(p_{c}\left(q^{\prime}\right), q^{\prime}\right)>\gamma\left(p_{c}(q), q\right)$, then by continuity there exists $p^{\prime}>p_{c}\left(q^{\prime}\right)$ and $p<p_{c}(q)$ such that $\gamma\left(p^{\prime}, q^{\prime}\right)>\gamma(p, q)$, in contradiction of (3.7). Now $\gamma$ is strictly monotone in both $p$ and $q$, and therefore $p_{c}(q)<p_{c}\left(q^{\prime}\right)$. 


\section{Disjoint occurrence}

In the study of percolation ([13]), the FKG inequality is complemented by the BK inequality. For a probability measure $\mu$ on $\Omega$, the latter inequality states that

$$
\mu(A \circ B) \leq \mu(A) \mu(B)
$$

for increasing events $A$ and $B$, where $A \circ B$ denotes the event that $A$ and $B$ 'occur disjointly'.

The formal definition of $A \circ B$ is as follows. Let $e_{1}, e_{2}, \ldots, e_{m}$ be the edges of the graph $G$. Any configuration $\omega(\in \Omega)$ is specified uniquely by the set $\eta(\omega)=$ $\left\{e_{i}: \omega\left(e_{i}\right)=1\right\}$ of edges having state 1 . We define the event $A \circ B$ to be the set of all $\omega$ for which there exists a subset $\psi$ of $\eta(\omega)$ such that $\omega^{\prime} \in A$ and $\omega^{\prime \prime} \in B$, where $\omega^{\prime}$ and $\omega^{\prime \prime}$ are determined by $\eta\left(\omega^{\prime}\right)=\psi, \eta\left(\omega^{\prime \prime}\right)=\eta(\omega) \backslash \psi$. We call $A \circ B$ 'the event that $A$ and $B$ occur disjointly', and note that $A \circ B$ is the set of configurations for which there exist disjoint sets of open edges with the property that the first set guarantees the occurrence of $A$ and the second guarantees $B$. A typical example of this definition is when $A$ and $B$ are 'connection events' of the type 'vertices $u$ and $v$ are joined by an open path'.

Clearly the BK inequality cannot hold in complete generality for random-cluster measures. For example, let $G$ be the graph with two vertices $u, v$, and two parallel edges $e, f$ each joining $u$ and $v$. With $A=\{\omega(e)=1\}$ and $B=\{\omega(f)=1\}$, we have that

$$
\phi_{p, q}(A \circ B)>\phi_{p, q}(A) \phi_{p, q}(B) \quad \text { if } 0<p<1, q>1,
$$

where $\phi_{p, q}$ is given in (1.1). One may ask about the possible validity of weaker forms of the BK inequality, of which the following is an instance.

Theorem 4.1. If $A$ and $B$ are increasing events and $q \geq 1$, then

$$
\phi_{p, q}(A \circ B) \leq \phi_{p, q}(A) \phi_{p, 1}(B)
$$

where $\phi_{p, q}$ is given by (1.1).

Note the presence of the product (percolation) measure $\phi_{p, 1}$ in the right-hand side of (4.2). Theorem 4.1 reduces to the usual BK inequality when $q=1$; see [3, $5,13,23]$. This theorem may be applied in various ways, but we have currently no interesting application which is not already available by use of comparison inequalities. Nevertheless we hope the inequality may have some interest in its own right. Inequalities of BK type are related in an intimate but mysterious way to the Hammersley-Simon-Lieb inequalities of mathematical physics (see [18, 20, 22]).

Proof. We present a sketch proof only, since a full proof would be unduly long. Our sketch is based on the 'duplication' argument of [3], as reworked in [13]. Let $G=(V, E)$ be a finite graph, and let $\phi_{p, q}$ be given by (1.1) where $0<p<1$ and $q \geq 1$.

The notation and details of the proof follow those presented on pp. 32-33 of [13]. We write $\Gamma=\{0,1\}^{E}, \mathcal{G}$ for the set of subsets of $\Gamma$, and consider the product space $\left(\Gamma_{1} \times \Gamma_{2}, \mathcal{G}_{1} \times \mathcal{G}_{2}, P_{12}\right)$ where $\Gamma_{i}=\Gamma, \mathcal{G}_{i}=\mathcal{G}$ for $i=1,2$, and $P_{12}=\phi_{p, q} \times \phi_{p, 1}$. The proof of [13] may now be followed with only one change. In that proof is constructed 
a mapping $\phi: \Gamma_{1} \times \Gamma_{2} \rightarrow \Gamma_{1} \times \Gamma_{2}$ which is a measure-preserving injection on a certain union $C_{1} \cup C_{2}^{\prime} \cup C_{2}^{\prime \prime}$ of events. In the current setting, $\phi$ remains an injection but is no longer measure-preserving; instead, it is measure-increasing in that

$$
P_{12}(\phi(x \times y)) \geq P_{12}(x \times y)
$$

for all relevant $x \times y$. Once (4.3) is established, the main inequality $((2.21)$ of [13]) follows as before, thereby completing the proof. Equation (4.3) is trivial if $x \times y \in C_{1} \cup C_{2}^{\prime}$, since $\phi$ is the identity map on this event. When $x \times y \in C_{2}^{\prime \prime}$, then $\phi(x \times y)$ is obtained by interchanging the values of $x_{k}$ and $y_{k}$. If $x_{k}=y_{k}$ then $\phi(x \times y)=x \times y$. Furthermore $x_{k}=1$ on $C_{2}^{\prime \prime}$, and so we need only consider the case $x_{k}=1, y_{k}=0$. For such $x, y$, we have that

$$
\frac{P_{12}(\phi(x \times y))}{P_{12}(x \times y)}=\left(\frac{p}{1-p}\right)\left(\frac{1-p}{p} q^{c\left(x^{\prime}\right)-c(x)}\right), \quad \text { if } 0<p<1,
$$

where $x^{\prime}$ is obtained from $x$ by setting $x_{k}=0$, and $c(z)$ is the number of components of a configuration $z\left(\in \Gamma_{1}\right)$; we have changed the notation here in order to remain as close as possible to the notation of [13]. Evidently $c\left(x^{\prime}\right) \geq c(x)$, and (4.3) follows when $0<p<1$. The result is trivially valid when $p=0,1$.

\section{Acknowledgements}

This work was aided by partial support from the Isaac Newton Institute, University of Cambridge, by the SERC under grant GR G59981, and by the EU under contract CHRX-CT93-0411. It is a pleasure to acknowledge Carol Bezuidenhout's suggestion of the possibility of proving the strict monotonicity of the critical point $p_{c}(q)$ as a function of $q$.

\section{References}

1. Aizenman, M., Chayes, J. T., Chayes, L., and Newman, C. M. (1988). Discontinuity of the magnetization in one-dimensional $1 /|x-y|^{2}$ Ising and Potts models. Journal of Statistical Physics 50, 1-40.

2. Aizenman, M. and Grimmett, G. R. (1991). Strict monotonicity for critical points in percolation and ferromagnetic models. Journal of Statistical Physics 63, 817-835.

3. Berg, J. van den (1985). Disjoint occurrences of events: results and conjectures. Particle Systems, Random Media and Large Deviations (R. T. Durrett, ed.), Contemporary Mathematics no. 41, American Mathematical Society, Providence, R. I., pp. 357-361.

4. Berg, J. van den and Fiebig, U. (1987). On a combinatorial conjecture concerning disjoint occurrences of events. Annals of Probability 15, 354-374.

5. Berg, J. van den and Kesten, H. (1985). Inequalities with applications to percolation and reliability. Journal of Applied Probability 22, 556-569.

6. Bezuidenhout, C. E., Grimmett, G. R., and Kesten, H. (1993). Strict inequality for critical values of Potts models and random-cluster processes. Communications in Mathematical Physics 158, 1-16.

7. Edwards, R. G. and Sokal, A. D. (1988). Generalization of the Fortuin-Kasteleyn-SwendsenWang representation and Monte Carlo algorithm. The Physical Review D 38, 2009-2012.

8. Fortuin, C. M. (1971). On the random-cluster model. Doctoral thesis, University of Leiden.

9. Fortuin, C. M. (1972). On the random cluster model. II. The percolation model. Physica 58, 393-418. 
10. Fortuin, C. M. (1972). On the random cluster model. III. The simple random-cluster process. Physica 59, 545-570.

11. Fortuin, C. M. and Kasteleyn, P. W. (1972). On the random cluster model. I. Introduction and relation to other models. Physica 57, 536-564.

12. Fortuin, C. M., Kasteleyn, P. W., and Ginibre, J. (1971). Correlation inequalities on some partially ordered sets. Communications in Mathematical Physics 22, 89-103.

13. Grimmett, G. R. (1989). Percolation. Springer-Verlag, New York.

14. Grimmett, G. R. (1994). Potts models and random-cluster processes with many-body interactions. Journal of Statistical Physics (to appear).

15. Grimmett, G. R. (1994). The stochastic random-cluster process, and the uniqueness of randomcluster measures (to appear).

16. Grimmett, G. R. (1994). The random-cluster model. Probability, Statistics and Optimisation (F. P. Kelly, ed.), John Wiley \& Sons, Chichester, pp. 49-63.

17. Grimmett, G. R. (1994). Percolative problems. Probability and Phase Transition (G. R. Grimmett, ed.), Kluwer, Dordrecht, pp. 69-86.

18. Hammersley, J. M. (1957). Percolation processes. Lower bounds for the critical probability. Annals of Mathematical Statistics 28, 790-795.

19. Kasteleyn, P. W. and Fortuin, C. M. (1969). Phase transitions in lattice systems with random local properties. Journal of the Physical Society of Japan 26, 11-14, Supplement.

20. Lieb, E. H. (1980). A refinement of Simon's correlation inequality. Communications in Mathematical Physics 77, 127-135.

21. Menshikov, M. V. (1987). Quantitative estimates and rigorous inequalities for critical points of a graph and its subgraphs. Theory of Probability and its Applications 32, 544-547.

22. Simon, B. (1980). Correlation inequalities and the decay of correlations in ferromagnets. Communications in Mathematical Physics 77, 111-126.

23. Talagrand, M. (1993). Some remarks on the Berg-Kesten inequality (to appear). 